\title{
DINAMIZACIÓN DE LAS RELACIONES FAMILIA-CENTRO ESCOLAR A TRAVÉS DE LA FORMACIÓN DEL PROFESORADO EN ESTE CAMPO DE ACTUACIÓN
}

\author{
ENHANCING FAMILY-SCHOOL PARTNERSHIPS THROUGH IN-SERVICE \\ TEACHER TRAINING PROGRAMS AND ACTION-RESEARCH IN THIS FIELD
}

\author{
Raquel-Amaya Martínez González; ; Marisa Pereira ${ }^{2}$; \\ Blanca Rodríguez Díez ; Ana Peña \\ Rosario Martínez ; $M^{a}$ Paz García González ${ }^{6}$; Begoña Donaire ${ }^{7}$; \\ Ana Isabel Álvarez ${ }^{8}$; Victoria Casielles ${ }^{9}$ \\ y Padres/Madres y personal Docente de los Centros implicados en el estudio \\ Universidad de Oviedo
}

\footnotetext{
1 Raquel-Amaya Martínez González. Doctora en Pedagogía. Profesora Titular de Universidad de Diagnóstico Pedagógico. Premio Nacional a la Investigación Educativa 1992. Sus líneas de investigación se centran en la Evaluación de Programas de Orientación Familiar, Evaluación de Necesidades Educativas Comunitarias y Escolares y Dinamización de la relación Familia-Centro Educativo. Ha participado en varios proyectos de investigación y formación nacionales e internacionales en el contexto norteamericano y europeo.

2 Marisa Pereira. Doctora en Pedagogía. Profesora Titular de Universidad de Orientación Profesional y Ocupacional. Premio Nacional a la Investigación Educativa 1992. Sus líneas de investigación se centran en la Orientación para la Carrera. Ha participado en varios proyectos de investigación y formación nacionales e internacionales en el contexto norteamericano y europeo.

3 Blanca Rodríguez Díez. Doctora en Pedagogía. Inspectora de Educación. Sus líneas de investigación se centran en la Evaluación de Centros Escolares. Ha participado en varios proyectos de investigación y formación.

4 Ana Peña. Doctora en Pedagogía. Profesora Asociada en el Departamento de Ciencias de la Educación de la Universidad de Oviedo. Sus líneas de investigación se centran en la Orientación de Alumno/as Sobredotado/as.

5 Rosario Martínez. Colegio La Milagrosa. Oviedo. Licenciada en Pedagogía. Profesora de Enseñanza Primaria y Secundaria. Doctoranda con el tema: Percepción de los padres y madres sobre la evaluación escolar.

6 Ma Paz García González. Colegio La Gesta. Oviedo. Licenciada en Pedagogía. Profesora de Educación Infantil.

7 Begoña Donaire Rubio. Becaria de Investigación. Universidad de Oviedo. Licenciada en Pedagogía.

8 Ana Isabel Álvarez. Becaria de Investigación. Universidad de Oviedo. Licenciada en Pedagogía.

9 Victoria Casielles. Becaria de Investigación. Universidad de Oviedo. Licenciada en Pedagogía.
} 


\title{
RESUMEN
}

En este artículo se describen algunas acciones llevadas a cabo para dinamizar las relaciones entre las familias y los centros escolares a través de la formación del profesorado en esta temática. Para ello se ha constituido un Seminario de Formación-Acción integrado por profesionales de la Educación que desarrollan su actividad en distintos niveles académicos (Directores/as y Profesores/as de centros escolares públicos y privados concertados de E.Infantil, Primaria, y Secundaria, una Inspectora de Educación, profesoras Universitarias y Licenciadas en Ciencias de la Educación). A través de la metodología de la Investigación-Acción Colaborativa se han configurado grupos de trabajo en los centros escolares, formados por padres/madres y profesores/as, que están permitiendo evaluar las necesidades que tienen los centros en materia de cooperación con las familias, y organizar actuaciones que den respuesta a dichas necesidades.

Palabras clave: Cooperación familia-centro escolar. Formación del Profesorado. Investigación-Acción.

\begin{abstract}
In this article the authors describe some actions carried out to develop family-school partnership through in-service training for teachers in this field. An Action-Training Seminar consisting of Professionals involved in different levels of education (Inspection, Teachers from Infant to Secondary Education, University Teachers and Post-graduate students in Education) has been set up to train primary and secondary school teachers and to help them build action-research teams with parents in schools. The aims of these teams based at the schools have been to analyze partnership needs and to organize some activities to meet them.
\end{abstract}

Key words: Family-school partnership. In-service teachers training. Action-research.

\section{La participación de la comunidad educativa como factor de calidad educativa}

La participación de la comunidad educativa en los centros escolares se ha revelado en múltiples estudios como un elemento importante de acercamiento y entendimiento entre padres y madres y profesorado y como una forma de enriquecer y facilitar el proceso de aprendizaje del alumnado, al aunar estas dos instancias sus esfuerzos educativos para la consecución de una meta común: la formación integral del alumno/a. Tal como han mostrado numerosos estudios, a través de la relación padres-centro escolar, los/as alumnos/as no solamente pueden llegar a elevar su nivel de rendimiento escolar, sino que, además, desarrollan actitudes y comportamientos positivos que, en definitiva, enriquecen su persona (Henderson, 1989; Balster, 1991; Hepwoth, 1991; Macbeth \& Ravn, 1994; Naciones Unidas, 1995; Johnson \& Goode, 1996; Martínez González, 1996; Martínez González y Corral Blanco, 1996; OCDE, 1997).

De otro lado, los estudios muestran que este nivel de relación es eficaz para controlar y prevenir el fracaso escolar, no sólo por el apoyo que los/as alumnos/as reciben de padres y madres y profesorado en su proceso de aprendizaje, sino también por la continuidad que perciben entre los objetivos educativos que se proponen en el ámbito familiar y los que se proponen en el ámbito escolar.

Esta es la conclusión a la que se llega en la mayoría de los estudios realizados sobre esta temática. Todos apuntan una idea común que engloba dos componentes relacionados: cuando los padres y madres participan activamente en el centro escolar, los/as hijos/as incre- 
mentan su rendimiento académico y, además, el centro mejora su calidad educativa, por lo que se considera que éstos son centros más eficaces en el desempeño de su labor formativa (Gordon, 1978; Leler, 1983; Becher, 1984, Davies, 1996).

Los procesos de participación que venimos mencionando tienen su cabida en el concepto de escuelas eficaces, que está generando gran interés, desarrollo y cambio en los centros escolares desde hace más de 20 años. La investigación sobre este campo ha puesto de manifiesto la existencia de muchos factores que contribuyen a generar escuelas eficaces, pero las conclusiones obtenidas no siempre son igualmente válidas en diferentes contextos. A la hora de identificar estos centros es importante tener en cuenta no sólo las características específicas de éstos como instituciones sino también la relación que mantienen estas características con los contextos donde los centros se encuentran interaccionando, lo que lleva a considerar la perspectiva ecológica en el estudio de los centros eficaces. En este sentido es importante tener en cuenta la implicación de las familias como factor que contribuye a su eficacia (Gordon, 1978; Phillips, et al., 1985; Epstein, 1987; Borrell, 1988; San Fabián, 1992; Tschorne at al., 1992; Martínez González, 1994, 1996).

Al mismo tiempo es preciso recordar los efectos positivos que esta implicación tiene también tanto para los padres y madres como para el profesorado y los/as alumnos/as (Martínez González, 1992, 1994a, b), lo que aconseja estimular y desarrollar una política educativa que permita formar a los/as profesores/as en estrategias de comunicación con las familias y que sea entendida como un medio de desarrollo de los centros y de incrementar la calidad de la educación. No obstante, a pesar de que está cada vez más reconocida la contribución de las familias en la génesis de eficacia en los centros, existe aún poca investigación sobre el tema que ayude a clarificar los procesos que la facilitan: cómo contribuyen los padres y madres al desarrollo de mecanismos de apoyo al profesorado, al incremento de medios en el centro a través de la presión tácita que ejercen, o a que los/as alumnos/as mejoren su rendimiento académico implicándose en su proceso de aprendizaje. Estas son cuestiones, entre otras, que precisan aún de una detallada investigación.

A pesar de los aspectos positivos comentados, algunos intentos de implementar programas de participación de los padres y madres en los centros han fracasado porque existen importantes diferencias actitudinales al respecto entre padres y madres y profesorado. En un estudio dirigido por Martínez González en Asturias (1994b) donde se analizan las necesidades de cooperación entre familias y centros, hemos encontrado que padres y profesores coinciden en manifestar lo siguiente:

«no se habla suficientemente sobre los objetivos educativos que se persiguen para los chicos, no se toman muchas decisiones juntos, no se elaboran planes conjuntos para solucionar problemas de los alumnos; se reúnen fundamentalmente para tratar problemas y no tanto para comentar aspectos positivos de los alumnos, y perciben que participan por igual en las actividades extracurriculares del centro. Pero en la mayoría de los casos, los profesores perciben que su comportamiento en las variables descritas se da con más frecuencia de lo que perciben los padres. De manera, que podríamos decir que en estos temas los padres son más exigentes que los profesores, o que les gustaría que se realizaran con más frecuencia de la que se realizan» (Martínez González et al, 1994: 173).

«En resumen, podríamos decir que cuando se trata de aspectos que tienen que ver con el grado de comunicación que existe entre padres y profesores para tratar objetivos educativos y organizar actividades y planes de actuación para mejorar situaciones de aprendizaje, los padres exigen más a los profesores; y éstos, por el contrario, exigen más a los padres cuando se 
trata de valorar comportamientos y actitudes educativas no tan ligadas a los procesos instructivos» (Martínez González et al., 1994b: 173).

En definitiva, los resultados de la investigación realizada sobre este tema apoyan y proclaman la necesidad de promover y favorecer la implicación de la comunidad educativa en los centros escolares como medio para mejorar no sólo la calidad de la educación que reciben los menores, sino también para propiciar el enriquecimiento profesional y personal de los/as profesores y padres y madres, y de incrementar la eficacia de los centros escolares.

El estudio que sigue a continuación pretende, precisamente, acercarse a esta finalidad a través del diseño de estrategias de formación del profesorado que permiten crear y fortalecer vínculos entre la familia y la escuela.

\section{Objetivos de la investigación}

El estudio se planteó con el objetivo fundamental de analizar algunos de los procesos que permiten promover la participación de la comunidad educativa en los centros escolares, especialmente de los padres y madres, a través de la investigación-acción como propuesta metodológica. Se trata de un proyecto que intenta estimular la innovación educativa en los centros escolares combinando la investigación, la acción y la formación del profesorado en materia de cooperación con la comunidad educativa.

Este objetivo general se ha concretado en dos objetivos específicos:

1. Estudiar la viabilidad de que se constituya en los centros escolares un equipo de trabajo formado por representantes del profesorado y de padres y madres, coordinado por un equipo investigador universitario, que permita analizar necesidades de cooperación entre los distintos sectores, y promover acciones encaminadas a fomentar la participación de la comunidad educativa en los centros escolares.

2. Evaluar las necesidades de cooperación entre las familias y el centro escolar en función de la tipología de actividades que se vienen promoviendo en los centros para facilitar la participación de la comunidad educativa.

\section{Metodología de investigación}

Se ha seguido una metodología basada en la investigación-acción cooperativa, acorde con la temática de estudio y con los objetivos de la experiencia.

\section{Diseño}

\section{Muestra}

Los centros participantes han sido auto-seleccionados en función del grado de estímulo, voluntariedad e interés en desarrollar la temática. Se trata de cuatro centros escolares de Asturias, tres públicos y uno concertado; uno de ellos es de Educación Especial. 


\section{Elementos personales y grupales}

a) Equipo de investigación de Base formado por:

1. Profesoras Universitarias adscritas al Departamento de Ciencias de la Educación de la Universidad de Oviedo,

2. una Inspectora de Educación,

3. Profesoras en ejercicio, que son, además, Licenciadas en Pedagogía, y

4. Licenciadas en Pedagogía.

b) Grupo de investigación-acción en los centros escolares, formado por representantes de padres y madres y profesores/as, entre los que se ha encontrado algún miembro del Equipo Directivo de los centros, ya fueran Directores/as, Jefes de Estudio o ambos a la vez. En todos los centros la estructura del grupo ha sido similar:

- Director/a

- una profesora de cada ciclo educativo

- padres/madres con hijos/as en los distintos ciclos educativos

- en algunas ocasiones, sobre todo al principio de la experiencia, alguna persona del equipo base estuvo también presente en algún centro cumpliendo una función de orientación y asesoramiento para facilitar el funcionamiento del grupo en estos primeros momentos.

\section{Procedimientos de recogida de información}

Dada la temática de estudio y la metodología a seguir, los procedimientos de recogida de datos han consistido en:

- Grupos de discusión a dos niveles: uno en cada centro escolar, configurado por padres/madres y profesores/as; y otro a nivel universitario con investigadores/as y representantes de todos los centros participantes en la experiencia, donde se pusieron en común los procesos y decisiones llevados a cabo en cada centro y se asesoró y orientó la actividad investigadora que se desarrolló en los mismos.

- Observación participante de:

- los procesos de participación de la comunidad educativa en los centros, y de los efectos que producen,

- las dinámicas que se desarrollan en los grupos de discusión con base en los centros y a nivel universitario.

- Análisis de Documentos Institucionales de los centros (Proyecto Educativo de Centro, Programación General Anual, Proyecto Curricular y Memoria Evaluativa Final) donde se puede identificar el nivel de participación que se concede a la comunidad educativa y los efectos que con ello se pretenden conseguir.

- Entrevistas y cuestionarios a padres y madres, profesores/as y alumnos/as que han permitido identificar factores que facilitan o dificultan la participación, sus efectos y las necesidades de cooperación que perciben distintos sectores de la comunidad educativa. 


\section{Técnicas de análisis de datos}

Básicamente cualitativas y basadas en procesos, de acuerdo con la información que han aportado los distintos procedimientos de recogida de información: Análisis de Contenido de las sesiones de discusión de los grupos, de documentos institucionales de los centros, y de entrevistas; Análisis Observacionales de las dinámicas de los grupos, de las actividades de participación de la comunidad educativa y de los efectos derivados.

\section{Resultados: procesos y factores de éxito de la experiencia}

\section{Implicación de los grupos de trabajo y de los centros escolares (objetivo 1)}

\section{Bases formativas previas}

Las tres primeras sesiones de trabajo de este Seminario de Formación-Acción se dedicaron a tratar los siguientes aspectos que han resultado imprescindibles para la continuación de la experiencia:

- la fundamentación teórica del tema, tomando como base los resultados de diversas investigaciones que señalan los efectos positivos que se derivan de la dinamización de las relaciones entre las familias y los centros escolares: entre otros, el incremento de la calidad educativa que se ofrece en uno y otro contexto, el aumento de satisfacción de las familias y la mejora del rendimiento de los alumnos/as.

- Otro de los aspectos tratados antes de la constitución de los grupos de trabajo en los centros fue la metodología de actuación que se seguiría en los mismos, basada en la Investigación-Acción, y las implicaciones asociadas al uso de esta metodología.

- Finalmente la directora del proyecto explicó los pasos a seguir para la formación de los grupos de trabajo en los centros escolares.

Durante este período que podríamos llamar de iniciación, siete colegios participaron en esta fase de la experiencia, con una asistencia irregular por parte de los profesores/as y miembros de los equipos directivos. Finalmente, el número de centros implicados quedó en los cuatro mencionados. Entre las dificultades que han impedido a los restantes centros escolares participar en este estudio de investigación-acción cooperativa se encuentran las siguientes:

- problemas de compatibilidad de horarios

- distancia a la Facultad de Ciencias de la Educación (algunos están ubicados en zonas rurales a cierta distancia de Oviedo),

- las personas de los equipos directivos están implicadas en diversas actividades derivadas de sus cargos que requieren su atención y presencia directa, impidiéndoles su participación sistemática a las sesiones de trabajo,

- la inestabilidad de los equipos directivos, que ha hecho variar su composición en algunos centros y provocado el desánimo de algunos/as profesores/as a seguir trabajando en éste y otros temas, 
- las relaciones a veces conflictivas entre las Asociaciones de Madres y Padres de Alumnos/as y el Equipo Directivo de los centros,

- la falta de interés por parte de la dirección de algunos centros, aunque los/as profesores/as estuvieran interesados/as en participar.

Acciones de los grupos que constituyen indicadores de éxito de la experiencia

Acciones del Equipo de Investigación de Base:

1. Informar y asesorar a equipos directivos y profesores/as sobre el modo de proceder para formar los grupos de investigación-acción en los centros y operar en ellos (actividad de innovación y formación).

2. Visitar los centros involucrados en la investigación a fin de hacer física su presencia en los mismos, recoger información observacional y cualitativa de primera mano y asesorar al grupo de investigación-acción en su propio contexto de acción.

3. Reunir periódicamente a los representantes de los diversos grupos de investigaciónacción con base en los centros escolares para intercambiar información sobre sus acciones, progresos, limitaciones, aprendizajes y futuras acciones. A través de estos encuentros los centros participantes en la investigación pudieron estimularse mutuamente y aprender de las acciones y resultados que cada uno estaba efectuando y encontrando. Permitió también el asesoramiento mutuo entre iguales.

4. Asesorar a los centros sobre recursos, materiales y temáticas de interés: reuniones con padres, disciplina, autoestima, procesos de comunicación, etc.

5. Analizar documentos y procesar datos para realizar los oportunos estudios cualitativos y cuantitativos de las variables analizadas.

Acciones del grupo de investigación-acción de los centros escolares:

1. Reunirse periódicamente en el centro para analizar el estado actual de la participación de la comunidad educativa y sus efectos sobre la calidad de la educación.

2. Reunirse periódicamente con el equipo de investigación de base y con los participantes de los diversos centros para poner en común y debatir acciones y temas de interés.

3. Clasificar las actividades de participación de la comunidad educativa en las diversas áreas propuestas por el equipo investigador de base, a fin de clarificar acciones y de facilitar la comparación y puesta en común entre centros.

4. Adaptar al centro cuestionarios que han permitido comenzar a analizar las necesidades de participación de la comunidad educativa.

5. Revisar documentos institucionales como el Proyecto Educativo y la Programación General Anual para analizar cómo se contempla la participación de la Comunidad Educativa en el centro e identificar los efectos que dicha participación produce en la mejora de la calidad educativa. 


\section{Evaluación de necesidades de cooperación entre las familias y los centros escolares (Objetivo 2)}

El análisis de las actividades que se promueven cotidianamente en los centros para facilitar la participación de la comunidad educativa permite efectuar una evaluación inicial de necesidades en esta temática. La tipología de actividades que desarrollan generalmente los centros escolares para promover la cooperación con las familias puede clasificarse en seis áreas fundamentales que han sido descritas por Epstein (1989) y Martínez González (1992):

Área I: El centro como fuente de ayuda a las familias

Área II: La familia como fuente de ayuda al centro

Área III: Colaboración de los padres y madres en el centro

Área IV: Implicación de los padres y madres en las actividades de aprendizaje de sus hijos/as en casa

Área V: Participación de los padres y madres en los órganos de gestión y decisión del centro

Área VI: Conexión y coordinación del centro y las familias con otras entidades comunitarias.

Se entiende que cada centro desarrollará más un tipo de actividades que otro según sean sus objetivos educativos, el tipo de alumnado y familias que acoge, el ambiente social y comunitario en el que está inmerso y otros muchos factores. Un modo de evaluar necesidades de cooperación entre las familias y los centros consiste en identificar qué áreas está dinamizando más cada centro y cuáles menos; para ello se pueden clasificar las actividades que se realizan a lo largo del curso académico en las áreas mencionadas. La comparación de actividades entre centros aparece en las tablas que siguen a continuación, que permiten identificar, además, qué áreas de cooperación se desarrollan más y cuáles menos en el conjunto de los centros: 
ÁREA I: El centro como fuente de ayuda a las familias.

\begin{tabular}{|c|c|}
\hline ACTIVIDADES & TEMÁTICA \\
\hline $\begin{array}{l}\text { * Tutoría: } \\
- \text { Con padres y madres } \\
\quad \text { (individual/colectiva) } \\
- \text { con niños/as } \\
\\
\quad \text { (centros: C.L.N.U) }{ }^{1}\end{array}$ & $\begin{array}{l}\text { - Atención a la diversidad (L.U) } \\
\text { - Alimentos, aseo, sueño (C) } \\
\text { - (colectiva)Cambios legislativos(U) } \\
\text { - Contacto con los padres y madres que son nuevos en el } \\
\text { centro (C.U) } \\
\text { - (colectiva)reuniones de información de principio de } \\
\quad \text { curso. (U.C) } \\
\text { - Visita de padres y madres a final de curso (U) } \\
\text { - Tutorías con padres y madres un día a la semana (N.C.U) } \\
\text { - Boletín de notas (U) } \\
\text { - Informes a las familias (C) }\end{array}$ \\
\hline * Equipo orientador (N.C.U) & $\begin{array}{l}\text { - Aplicación Tests psicotécnicos }(\mathrm{U}) \\
\text { - Trabajadora social una vez cada quince días }(\mathrm{N})\end{array}$ \\
\hline * Escuela de padres y madres (U) & Relaciones padres-hijos/as (U) \\
\hline * Información impresa & $\begin{array}{l}\text { - Circulares informativas (U.N.C) } \\
\text { - Periódico escolar (U,C) }\end{array}$ \\
\hline
\end{tabular}

${ }^{1} \mathrm{U}=$ Colegio Concertado Ursulinas; L = C.P. La Luna; C = C.P. Ed. Especial Castiello, N = C.P. Noega.

ÁREA II: La familia como fuente de ayuda al centro.

\begin{tabular}{|c|}
\hline ACTIVIDADES \\
\hline$*$ Información médico-sanitaria y neurológica (C), en alumnos/as con \\
necesidades educativas especiales (U)
\end{tabular}


ÁREA III: Colaboración de los padres y madres en el centro.

\begin{tabular}{|c|c|}
\hline ACTIVIDADES & TEMÁTICA \\
\hline * Acompañar en las salidas (L) & $\begin{array}{l}\text { - natación }(U) \\
\text { - excursiones }(U)\end{array}$ \\
\hline * AMPAs & $\begin{array}{l}\text { - sola: } \\
\quad \text { - apoyo económico (C.U) } \\
\quad \text { - charlas y conferencias(C.U) } \\
\text { - canalización de problemas(N) } \\
\quad \text { - organización actividad extraescolar. (N.L.U) } \\
\text { - viajes de estudios }(\mathrm{N}) \\
\text { - concursos }(\mathrm{N}) \\
\text { - en colaboración con el centro: } \\
\text { - fiestas y festivales, teatro (N.C.L.U) }\end{array}$ \\
\hline $\begin{array}{l}\text { * Monitores de talleres de actividades } \\
\text { extraescolares (N.U) }\end{array}$ & \\
\hline $\begin{array}{l}* \text { Visitas culturales para conocer los } \\
\text { trabajos de los padres y madres }(\mathrm{U})\end{array}$ & \\
\hline * Mejora de infraestructuras (N.U) & \\
\hline $\begin{array}{l}\text { * Información de las profesiones de los } \\
\text { padres y madres }(U)\end{array}$ & \\
\hline
\end{tabular}

\section{ÁREA IV: Implicación de los padres y madres en las actividades de aprendizaje de sus hijos/as en casa.}

\begin{tabular}{|l|l|}
\hline \multicolumn{1}{|c|}{ ACTIVIDADES } & \multicolumn{1}{c|}{ TEMÁTICA } \\
\hline * Información a padres y madres cuyos/as \\
$\begin{array}{l}\text { hijos/as tienen dificultades de aprendizaje sobre } \\
\text { pautas a seguir en casa para apoyar y reforzar } \\
\text { este aprendizaje (L.C.N.U) }\end{array}$ & $\begin{array}{l}\text { - Elaboración de materiales (C.U) } \\
\text { - Elaboración de un calendario de } \\
\text { actividades de uso doméstico (C.U) } \\
\text { - Elaboración de paneles de conducta (C.U) }\end{array}$ \\
\hline * Orientación sobre técnicas de estudio (U) & \\
\hline
\end{tabular}


ÁREA V: Participación de los padres y madres en los órganos de gestión y decisión del centro.

\begin{tabular}{|l|l|}
\hline \multicolumn{1}{|c|}{ ACTIVIDADES } & \multicolumn{1}{c|}{ TEMÁTICA } \\
\hline * Consejo escolar (U.C.L.N.) & $\begin{array}{l}\text { - Comisión de Convivencia (C.L.) } \\
- \text { Comisión Económica (L) } \\
\text { - Comisión Actividades Extraescolares (L) }\end{array}$ \\
\hline * Reuniones semanales con la directora (N) & \\
\hline * Asambleas (N) & \\
\hline * Proceso de evaluación del centro (N.U) & \\
\hline * Consulta sobre temas generales (N) & \\
\hline
\end{tabular}

\section{ÁREA VI: Conexión y coordinación del centro y las familias con otras entidades comunitarias.}

\begin{tabular}{|c|}
\hline ACTIVIDADES \\
\hline Recogida de papel (U) \\
\hline Campañas (U) \\
\hline Ayuntamiento, Principado (L.N.U.C) \\
\hline Sanidad, Centros de Salud (L.C.U) \\
\hline Universidad (N.C) \\
\hline Otras AMPAs (N) \\
\hline Centro cultural del barrio (N) \\
\hline Centros ocupacionales (C) \\
\hline Cruz Roja (C.U) \\
\hline Equipos Psicopedagógicos, Trabajadora social (N.C) \\
\hline MEC (N) \\
\hline ONCE (C) \\
\hline Dirección General de Tráfico (U) \\
\hline
\end{tabular}

Del análisis efectuado se desprende que el área I: «El centro como fuente de ayuda a las familias» es el que incluye más actividades de relación con los padres y madres, que se concretan, sobre todo, en las tutorías y en aquellas otras que están legisladas, y que por tanto, son de obligado cumplimiento, como es el caso también de la participación de los padres y madres en el consejo escolar del área V; no se han identificado en esta área I actividades no legisladas que pudieran surgir como iniciativa propia de los centros. Por otra parte, las actividades que se realizan en cada uno son variadas y pocas veces coinciden varios centros en desarrollar las mismas. Esta diversidad de actuaciones resulta interesante porque a partir de 
ella los centros pueden incorporar algunas actividades que ya están realizando otros con éxito.

Esta tarea de revisión de actividades de cooperación entre el centro y las familias y de su clasificación en áreas para poder analizarlas mejor es importante porque permite identificar en cuáles cada centro está trabajando más y menos y detectar necesidades de actuación.

Tras este análisis de necesidades se pueden efectuar propuestas de actuación en las que cada centro se fije como meta para trabajar durante el curso siguiente mejorar algunos aspectos que tienen más descuidados en las áreas mencionadas.

\section{Conclusiones e implicaciones educativas}

Los procesos realizados a lo largo de la experiencia efectuada llevan a establecer algunas conclusiones que reflejamos a continuación.

El constituir un grupo de trabajo en los centros formado por padres y madres y profesore/as para analizar el estado de la participación y cooperación entre ambos es un proceso laborioso que requiere de ajustes y reajustes que conllevan un tiempo largo y la necesidad de superar momentos de desmotivación. Sin embargo, la configuración de este grupo de actuación basado en la investigación-acción es posible y una vez establecido, los profesores/as participantes en la experiencia han informado que permite alcanzar metas educativas más elevadas y contribuye a generar un alto grado de satisfacción por el trabajo realizado. Estos resultados valorativos son acordes con los descritos en la investigación realizada sobre esta temática, reseñada en la primera parte de este artículo.

Las actividades analizadas y su clasificación en áreas ha permitido observar que las oportunidades de participación de los padres y madres en los centros escolares son, en ocasiones, mayores de lo que inicialmente se percibe cuando este análisis no se ha realizado, lo que indica la conveniencia de sistematizar y recoger por escrito las actividades que se realizan en un campo determinado de actuación. Al mismo tiempo, esta sistematización ha permitido observar que existen áreas de cooperación en las que los centros apenas desarrollan actividades conjuntamente con los padres y madres, y que, por tanto, necesitan ser mejoradas y desarrolladas; se trata, fundamentalmente, de actuaciones vinculadas a los aspectos curriculares. A este respecto, los centros participantes han planteado ya iniciativas de mejora. Por el contrario, la colaboración en actividades extracurriculares es la más desarrollada en todos los centros.

Los contactos entre padres y madres y profesorado se efectúan con más frecuencia cuando el motivo de acercamiento es una situación problemática que afecta a los hijos/as que cuando se trata de comentar aspectos positivos. Esta situación podría influir en la calidad de las relaciones que se establecen entre ambos, dado que las situaciones problemáticas, por sí solas, tienden a generar actitudes negativas, enfrentamientos, tensiones y sentimientos de culpabilidad. De ello se deriva la conveniencia de conjugar en los encuentros de padres y madres y profesores/as el tratamiento de los problemas con el de aspectos positivos de los hijos/as que generen también satisfacciones.

Por otra parte, hemos observado en esta experiencia que los padres y madres cuyos/as hijsos/as acuden a centros públicos ordinarios que tienen estudios medios y universitarios 
tienden a tomar por sí mismos la iniciativa de contactar con el profesorado con más frecuencia de lo que lo hacen los padres y madres que poseen estudios primarios. Esto podría indicar que el nivel de estudios de los padres y madres influye en el número de contactos que establecen con los profesores/as para informarse de los progresos y circunstancias académicas de sus hijos/as, lo que les facilitará realizar un mejor seguimiento de su proceso de aprendizaje. De ello se deriva la conveniencia de que los centros estimulen a los padres y madres que tienen menores niveles de estudios a que contacten con el profesorado y a que se impliquen en las actividades de seguimiento de los progresos de sus hijos/as.

No obstante, a lo largo del proceso de realización de la experiencia hemos observado que no siempre le resulta fácil y posible al profesorado realizar estas acciones de estimulación de la participación y apoyo a las familias. En ocasiones, los/as profesores/as han comentado la falta de prepación al respecto, de lo que se deriva la necesidad de formación del profesorado y de los profesionales de la educación en general en habilidades y estrategias de comunicación con los padres y madres que les permitan estimular la colaboración con las familias de un modo más eficaz para incrementar la calidad educativa que reciben los menores.

Este ha sido, precisamente, el objetivo último que ha guiado el proceso de ejecución de la experiencia realizada en este Seminario de Formación-Acción que seguimos desarrollando en la Facultad de Ciencias de la Educación de la Universidad de Oviedo.

\section{Bibliografía}

BALSTER, L. (1991): Involving at-risk families in their children's education. ERIC Clearinghouse of Educational Management.: University of Oregon.

BECHER, R. (1984): Parent involvement: A review of research and principles of successful practice. National Institute of Education. Washington, D.C.

Borrell, N. (1988): Evaluación de centros escolares. En R. Pascual (Ed.). La gestión educativa ante la innovación y el cambio. II Congreso Mundial Vasco. Madrid, Narcea.

Davies, D. and Johnson, V. (Eds) (1996): Crossing Boundaries. Multi-National Action Research on Family-School Collaboration. Boston: Center on Families, Communities, Schools \& Children's Learning.

Epstein, J. (1987): Parent involvement. What research says to administrators. Education and urban society. Vol. 19(2): 119-294.

Epstern, J. (1989): Parents's attitudes and practices of involvement in inner-city elementary and middle schools.Documento presentado al Annual Meeting of the American Educational Research Association, San Francisco California.

GoRDOn, I. (1978): What does research says about the effects of parents involvement on schooling? Documento presentado al Annual Meeting of the Association for Supervision and Curriculum Development.

Henderson, A. (1989): The evidence continues to grow. Parent involvement improves student achievement. Columbia, Maryland, National Committee for Citizens in Education.

HePworth Berger (1991): Parents as partners in education. The school and home working together. New York, Macmillan.

Johnson, V. \& Goode, D. A. (1996): The community portrait: Promoting images through school, parent, and community collaboration. Forum of Education, 51(1), 23-34 
LELER, H. (1983): Parent education and involvement in relation to thr schools and to parents of school-aged children, en R. Haskins and D. Addams (Ed.). Parent education and public policy, N.J. ABLEX Publishing Co.

Macbeth, A. and Ravn, B. (Eds.) (1994): Expectations about parents in education. European Perspectives. University of Glasgow.: European Parents' Association.

Martínez GonZÁlez, R. A. (1992): Exploración diagnóstica de las necesidades de cooperación entre familia y centro escolar, Entemu: 63-80. Gijón, Centro Asociado de la UNED en Asturias.

Martínez GonzÁlez, R. A., Marques, R., and Souta, L. (1994a): Expectations about parents in Education in Portugal and Spain, in A. Macbeth and B. Ravn, Expectations about parents in education. Europeran Perspectives. University of Glasgow, European Parents' Association.

Martínez GonzÁlez, et al. (1994b): Diagnóstico de necesidades en la cooperación entre familia y centro escolar. Informe de Investigación inédito. Universidad de Oviedo.

Martínez GonzÁlez, R. A. (1996): Parent involvement in schools in Spain: A case study, en D. Davies and V. Johnson (Eds). Crossing Boundaries. Multi-National Action Research on FamilySchool Collaboration. Boston, Center on Families, Communities, Schools \& Children's Learning.

Martínez GonzÁlez, R. A. (1996): Familia y Educación. Fundamentos teóricos y metodológicos. Servicio de Publicaciones de la Universidad de Oviedo.

Martínez González, R. A. and Corral Blanco, N. (1996): The need of partnership: A comparison of parents and children in Spain, Forum of Education, 51(1): 73-82.

Naciones Unidas (1995): Families and Education. Occasional Papers Series, N 18.

OCDE (1997): Parents as partners in schooling. París, Centre for Educational Research and Innovation.

Phillips, S., Smith, M. \& Witte, J. (1985): Parents and schools. Staff report to the study Commission on the Quality of Education in the Metropolitan Milwaukee.

SAN FABIÁN, J. L. (1992): Gobierno y participación en los centros escolares: sus aspectos culturales. En Grupo de Investigación Didáctica (Comp.). Cultura escolar y desarrollo organizativo. Sevilla, Kronos.

Tschorne, P., Villalta, M. y Torrente, M. (1992): Padres y madres en la escuela. Una guía para la participación. Barcelona, Paidós.

Fecha de recepción: 17-5-99

Fecha de revisión: 18-10-99

Fecha de aceptación: 11-4-00 